\title{
Corneal Surgery (with DVD-ROM), Zuberbuhler B, Tuft S, Gartry D, Spokes D, (2013), ISBN: 978-3-642-12501-0, Springer
}

\author{
Claus Cursiefen
}

Received: 25 June 2014 / Accepted: 30 June 2014 / Published online: 23 July 2014

(C) Springer-Verlag Berlin Heidelberg 2014

Corneal Surgery is a joint venture by British cornea specialists from Moorfields Eye Hospital, St. Thomas Hospital in London, and St. James University Hospital in Leeds. The authors provide a portable and condensed overview of the current surgical techniques in cornea and external eye disease. They do not aim to provide an extended textbook and also their aim is not to present cutting-edge new procedures but rather commonly available and established surgical techniques.

The authors succeeded in providing a very condensed overview of all commonly performed surgical techniques in corneal surgery. The omission of all references and exhaustive discussions allows them to keep the textbook in an acceptable and portable format.

The book starts with an introduction into anatomy and common surgical procedures as well as corneal examination techniques. All modern examination techniques such as OCT, topography, and wavefront systems are well covered.

They then describe all common surgical techniques for ocular surface and reconstructive surgery such as amniotic membrane transplantation, superficial keratectomy, and PTK.

The next large chapter covers discussions on eye banking, penetrating, as well as all modern lamellar surgical transplant approaches. Here, DSAEK, DMEK, as well as DALK are well covered.
The last large chapter covers corneal refractive surgical procedures focusing on LASIK, LASEK, PRK, and also introducing all the common laser platforms available.

Very helpful for the budding corneal surgeon is the last section of the book, which is a wet laboratory introduction and description, which is accompanied by a DVD explaining the essential surgical techniques in corneal surgery.

In my opinion, this handy textbook is a very well condensed and concise overview of the most commonly performed surgical techniques in corneal and refractive surgery. It will be very helpful for the budding corneal surgeon, the corneal and refractive surgery fellow, and also as a short reference book for corneal surgeons. Since references are missing, the presented evidence is somewhat subjective, and also in certain aspects reflecting more the common practice pattern in the UK. These are not always representative for other European countries, which seems to be especially true for the modern surgical procedures such as a corneal crosslinking, DMEK, and DALK.

In summary, I think this condensed and well-pictured essential surgical technique guide will be helpful for budding corneal surgeons and is a quick reference guide for more experienced ophthalmic surgeons with a cornea focus.
C. Cursiefen $(\square)$

Department of Ophthalmology, University of Cologne, Cologne, Germany

e-mail: claus.cursiefen@uk-koeln.de 